\title{
Tunable Optical Filters for Space Exploration
}

\author{
Charles Crandall, High Chiva, Tucson AZ \\ Natalie Clark and Patti Davis NASA LaRC, Hampton Virginia, 23681
}

\begin{abstract}
Spectrally tunable liquid crystal filters provide numerous advantages and several challenges in space applications. We discuss the tradeoffs in design elements for tunable liquid crystal birefringent filters with special consideration required for space exploration applications. In this paper we present a summary of our development of tunable filters for NASA space exploration. In particular we discuss the application of tunable liquid crystals in guidance navigation and control in space exploration programs. We present a summary of design considerations for improving speed, field of view, transmission of liquid crystal tunable filters for space exploration. In conclusion, the current state of the art of several NASA LaRC assembled filters is presented and their performance compared to the predicted spectra using our PolarTools modeling software.
\end{abstract}

Key Words: Tunable filters, birefringent, Fabry Perot Etalon

\subsection{Introduction}

Tunable filters play in important role in many optical systems. Our LaRC cleanroom has the capability of fabricating a wide range of tunable optical filters. One category of tunable filters uses fixed parallel plates and tunes the refractive index of an electro-optic material that fills the cavity between the plates. When a voltage is applied across these plates the refractive index of the intervening layer changes. In this paper we describe the design and fabrication of such tunable filters that use birefringent materials. A second sensor category of tunable filters we also discuss are Fabry-Perot filters based on a new compliant based material. These compliant based Fabry-Perot Etalons have many advantages over the more common Piezo controlled variety. Typical piezo actuated Fabry-Perot interferometers have employed silicon MEMS technologies to control the separation between the plates. This type of device typically has difficulty maintaining the necessary tight tolerance on the parallel orientation of the plates. Doing so is complex and expensive. Furthermore, piezo devices, in addition to requiring high voltages, have a relatively narrow operating temperature range. The voltage and thermal requirements of the piezo-electric devices impose difficult and labor-intensive manufacturing and assembly operations, adding cost to the devices and making them less reliable. Our compliant polymer based compliant material Fabry-Perot based etalons offer the advantage of being able to be controlled with lower voltages and over a wider temperature range making the more suited for space exploration applications.

\subsection{Classical Birefringent filters}

The underlying concept in spectral filters made with birefringent media is that a single polarization state can be made to interfere with itself. In fact, many refer to these devices as Polarization Interference Filters or simply PIF filters. In a birefringent media this is accomplished by dividing the input wave into two orthogonal polarization states that propagate along the fast and slow axes of the birefringent media. Because of the differing indices of refraction one component is delayed relative to the other. Once the waves exit the media they are recombined and allowed to interfere. The birefringence, orientation and thickness of the media determine the spectral profile of the exiting wave.

The pioneering work pursued by both Bernard Lyot in the 1920's and later by Ivan Solc in the early 1950's was motivated by the desire of astronomers to analyze the spectral emissions of celestial bodies to determine their chemical makeup and thermal properties. Lyot himself used his invention to study the chromosphere of the sun and to prove the existence of water in the viscous atmosphere of Venus. ${ }^{1-2}$

Lyot filters are made with multiple stages of birefringent crystals sandwiched between successive polarizing elements. Lyot aligned the fast axis of calcite, a uniaxial birefringent element, parallel to the surface and $45^{\circ}$ relative to the transmission axes of the polarizers. Although Lyot used cascaded birefringent elements in the ratios 1:2:4: ... 2n, subsequent modifications of his technique have used different ratios and additional elements to adjust characteristics such as contrast. The more stages in a Lyot filter, the larger the finesse ratio and higher the discrimination power of the filter. However, due to the need for the intervening polarizer, the filter’s optical transmission deteriorates markedly with each additional stage.

The spectral transmission for the classical Lyot filter ${ }^{1}$ with $\mathrm{n}$ elements is 


$$
T(\lambda)=\frac{1}{4^{n}} \frac{\sin ^{2}\left(\frac{2^{n} \pi d \Delta n(\lambda)}{\lambda}\right)}{\sin ^{2}\left(\frac{\pi d \Delta n(\lambda)}{\lambda}\right)},
$$

In which $\lambda$ is the wavelength, $d$ is thickness of the shortest element and $\Delta n(\lambda)$ is the wavelength dependant birefringence of the media.

In 1953, Ivan Solc extended Lyot's work, constructing a filter that had several birefringent elements sandwiched between only two polarizers. By carefully orienting several retardation plates of equal thickness between two polarizers in either a fan or folded configuration, Solc traded improved transmission for a slight loss of spectral purity. The orientation angle that the fast axis makes with the horizontal of plate $k$ in an $n$ element Solc fan design is give by,

$$
\alpha_{k}=\frac{2(k-1) \pi}{4 n}
$$

Similarly, the orientation angles of the elements in the folded variety are alternating $+\pi / 4 n$ and $-\pi / 4 n$ radian angles, where $n$ is an even integer number of cells.

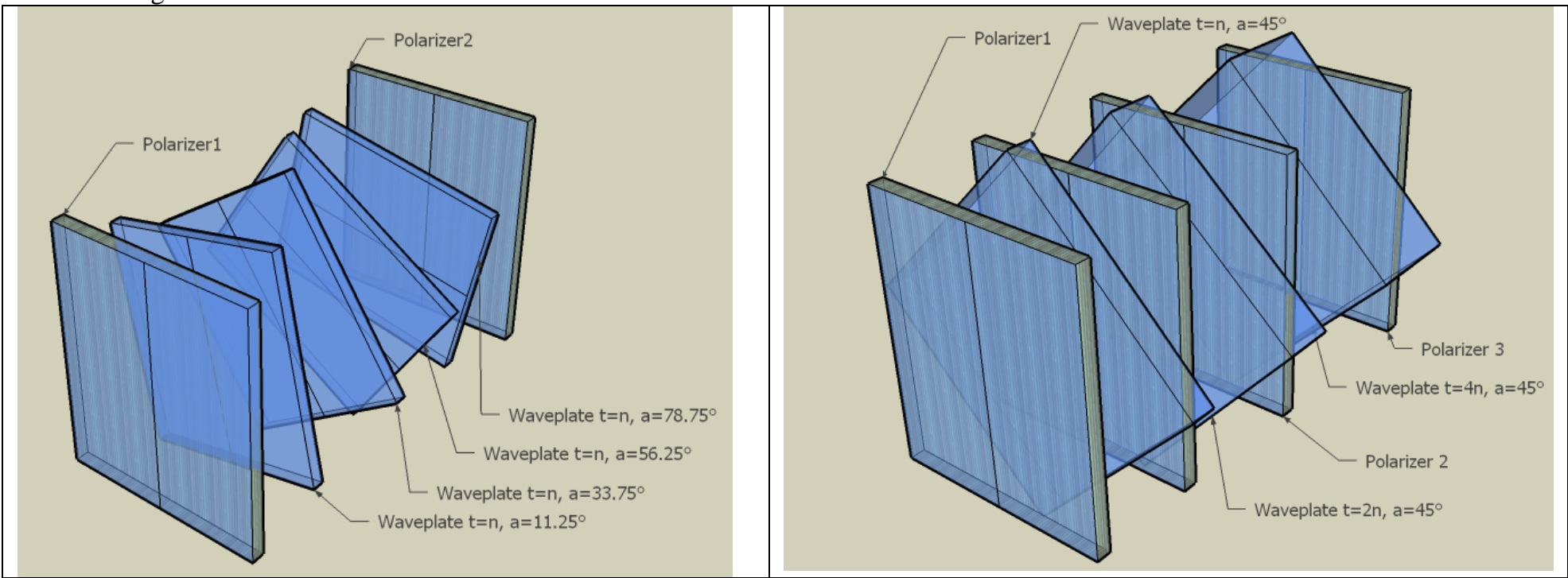

Figure 1. 3D Assembly illustrations of 4 stage Solc and 3 stage Lyot type filters in which the root wave pate retardance is $\mathrm{n}$ units and a is the fast axis orientation with respect to the horizontal.

It has been shown elsewhere ${ }^{4}$ that the spectral transmission of an $n$ plate Solc filter can be calculated as

in which

$$
T(\lambda)=\left[\frac{\sin n \chi}{\sin \chi} \cos \chi \tan \alpha\right]^{2},
$$

$$
\cos \chi=\cos \left(\frac{\pi d \Delta n(\lambda)}{\lambda}\right) \cos \alpha
$$

and $\alpha$ is $\pi / 4 n$ radians. 
For comparison, the graphs below display the relative spectral transmission profiles of a three stage Lyot and a four stage Solc fan filter. The root media for each filter is birefringent polycarbonate sheet with a retardance of $2000 \mathrm{~nm}$ at $550 \mathrm{~nm}$, or just less than 4 waves. The three stage Lyot filter is made with a $2000 \mathrm{~nm}$, a $4000 \mathrm{~nm}$ and an $8000 \mathrm{~nm}$ stages separated by parallel polarizers- 4 in total. The fast axis of all elements is oriented $45^{\circ}$ to the transmission axis of the polarizers. By contrast, the Solc filter is made by arranging four $2000 \mathrm{~nm}$ birefringent sheets between parallel polarizers with fast axis alignment at the following angles: $11.25^{\circ}, 33.75^{\circ}, 56.25^{\circ}$ and $78.75^{\circ}$.

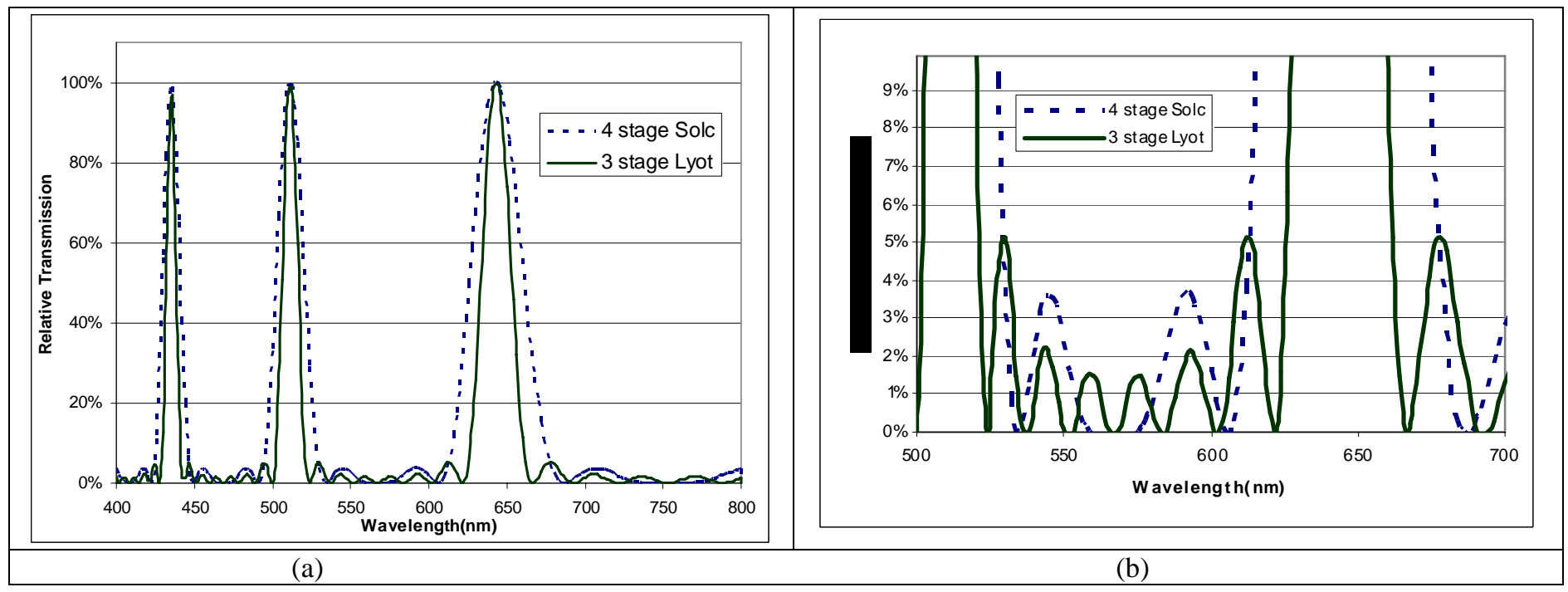

Figure 2. Comparison of the spectral transmissions for the example Lyot filter and Solc filters. (a) shows the full visible spectrum of a 3 stage Lyot and a 4 stage Solc filter and (b) show the detail of the side lobes.

As the root retardance of the birefringent element is $2000 \mathrm{~nm}$, one would anticipate a spectral peak at 500nm as that would be the integer four wave peak. It is because of the non-negligible dispersion of the birefringence that shifts this peak to a higher wavelength.

Neglecting the thickness of the polarizers, the optical thickness of the Lyot filter is $14 \mu \mathrm{m}$ whereas that of the Solc filter is only $8 \mu \mathrm{m}$. The added retardance of the Lyot cell accounts for the narrower spectral peaks, but the requisite additional two polarizers reduce the relative maximum throughput by no less than $70 \%$. Notice the larger secondary lobes of the Solc filter. These can be lessened with a slight precise angular variance in rotation of the birefringent plates.

\subsection{Tunable Spectral Filters}

The addition of liquid crystal (LC) variable wave plates to each stage of the filters allows spectral peaks to be dynamically tuned. Liquid crystal devices are fabricated of two specially coated glass substrates separated by spacers several microns in size and filled with liquid crystal. The LC is typically aligned at the surfaces by a rubbing a spun coated then cured polyamide or similar polymer. The voltage across the cell is delivered using an AC signal applied to the inner transparent conducting coated glass substrates. This amplitude of the voltage changes the alignment of the molecules in the center of the cell while those at the surface are fixed and supply a restoring torque.

Typical designs employ an anti-parallel buffed nematic state liquid crystal cell fixed to each static wave plate shim to construct a device with voltage controlled bounded retardance range. In the first order design the thickness of each cell should vary proportionally to the static shim retardation value. Consequently, to fabricate a tunable Lyot as described above one would need to use three liquid crystal devices of thickness $t, 2 t$ and $4 t$, where $t$ is the LC spacing thickness, which is directly proportional to the spectral tuning range. To modify the Solc example, four cells of equal thickness can be used aligned with or in place of the static shims.

\subsection{Tradeoffs}

Constructing tunable filters with tunable LC components introduces several second order effects that must be careful considered. For instance, once a voltage is applied across the cell, the liquid crystal molecules adjust to a new position. This is analogous to tipping a wave plate about the fast axis. As a result the field of view of the cell changes with voltage. This can be mitigated with a wide-fielding technique (such as by using two opposite tipping LC cells separated by a properly aligned half wave plate) at the expense of overall filter thickness, complexity and cost. As liquid crystals are a state of matter more akin to 
a liquid than to a solid, one would be correct in assuming that thermal effects also cannot be ignore. Though beyond the scope of this presentation, reference 6 has a wonderfully detailed treatment of waveplate field-of-view and temperature effects.

Filter performance criteria vary by application. Filter design requires trading off discrimination power against optical throughput. Discrimination power is expressed as the finesse of the filter, calculated as the ratio of the separation between the spectral peaks of the filter to the bandwidth of the peaks. The larger the finesse, the narrower and more widely separated the spectral peaks. High finesse ratios and low optical throughput are typical of the passive, multistage birefringent filters pioneered by Lyot and Solc.

With the development of lasers, there was increased interest in optical networks with highly precise filters. Extending the work of Lyot and Sole by applying Fourier approximation techniques, S. E. Harris et al documented a procedure for developing filters to produce any desired transfer function that is periodic with frequency and whose corresponding impulse response is real. ${ }^{3}$ For example, using this technique, it is possible to build a filter with the sharp spectral purity of a Lyot filter and the improved transmission of a Solc device. Similar to the Solc filters, these filters consisted of cascaded birefringent crystals between input and output polarizers. Though quite mathematically complex, this technique has been refined to fabricate small filters with very flat channel passbands to be used for wavelength-division multiplexers in the near-IR. ${ }^{4}$

\subsection{Virtual Prototyping Approach}

An alternative approach used to model and study birefringent devices has been developed by High Chiva Systems in conjuncture with NASA Langley Research Center (LaRC). Using PolarTools, a polarization library for LabVIEW, software utility (named FilterPRO) was developed that allows the user to select, add and easily manipulate birefringent devices to view both the resulting spectra and elliptical polarization state.

FilterPRO employs a database of real birefringent media index and dispersion parameters to accurately model the spectral polarization characteristics of any arbitrary on-axis multi-element devices. An intuitive user interface allows the user to scan through the chosen spectrum and explore the polarization characteristics at any intermediate point inside a multi-element device. The mathematical construct behind FilterPRO is a self indexing Meuller matrix multiplication scheme. For this reason, FilterPRO can be used for any birefringent element for which a Meuller matrix can be written. This can include cholesteric LC's, biaxial media, or even organic materials with rotary power (like sugar solutions) etc. The current database includes dispersion characteristics for several commercially available nematic liquid crystals, ferroelectrics and a number of static materials. Adding new materials to the database is straightforward. Designing complex polarization controlling devices in this manner allows the user to build an understanding of what is actually happening to the polarization states in between elements and therefore, refine designs as necessary. It is possible to save and load designs as desired.

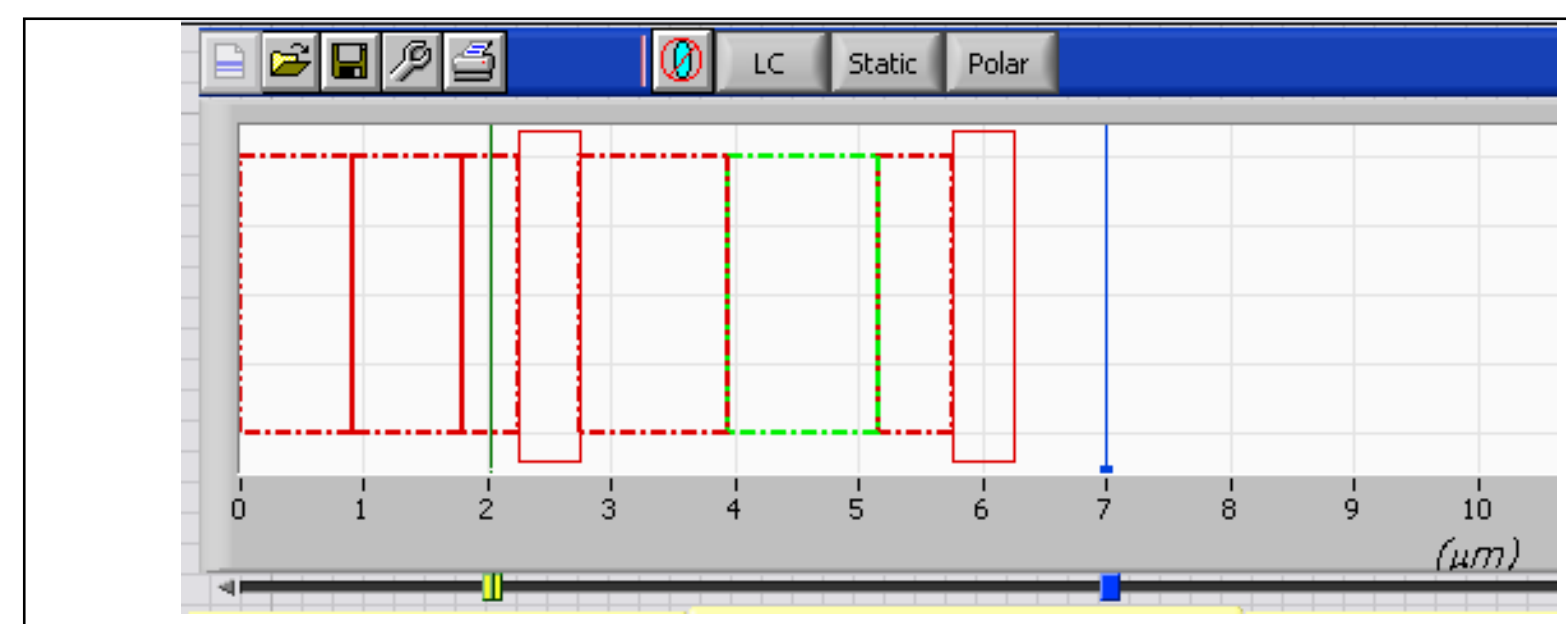

Figure 3. Layout area of Filterpro showing toolbar and cursors. Dashed items are static waveplates, solid items are polarizers. The cursors are used to examine intra-filter polarization states. The green element is active and can be manipulated.

The FilterPRO menu item selection or toolbar buttons are used to insert polarization elements. Once a selection has been made a subroutine scans the material database for conforming media and a window appears directing the user to input the remaining necessary parameters such as thickness, fast axis orientation angle etc. to describe the desired component. Ensuing elements are 
then displayed in a layout area graph where they can be selected altered and deleted. Cursors are used to probe polarization states at any point inside the layout. This approach facilitates virtual prototyping of tunable filters for advanced complex optical systems needed for NASA Space Exploration missions.

Below this graph FilterPRO displays useful details about the components and device as a whole. The polarization states both intra-device and as a complete unit can be displayed. Sub-components can be rotated, their thickness can be changed and their composition can be replaced with a different media. This allows the user to run numerous "what-if" scenarios and tolerancing before the real device is built. For more meaningful results, FilterPRO also calculates the spectral outputs can then be multiplied by the measured spectral transmission of the glasses, coatings and real polarizer throughputs.

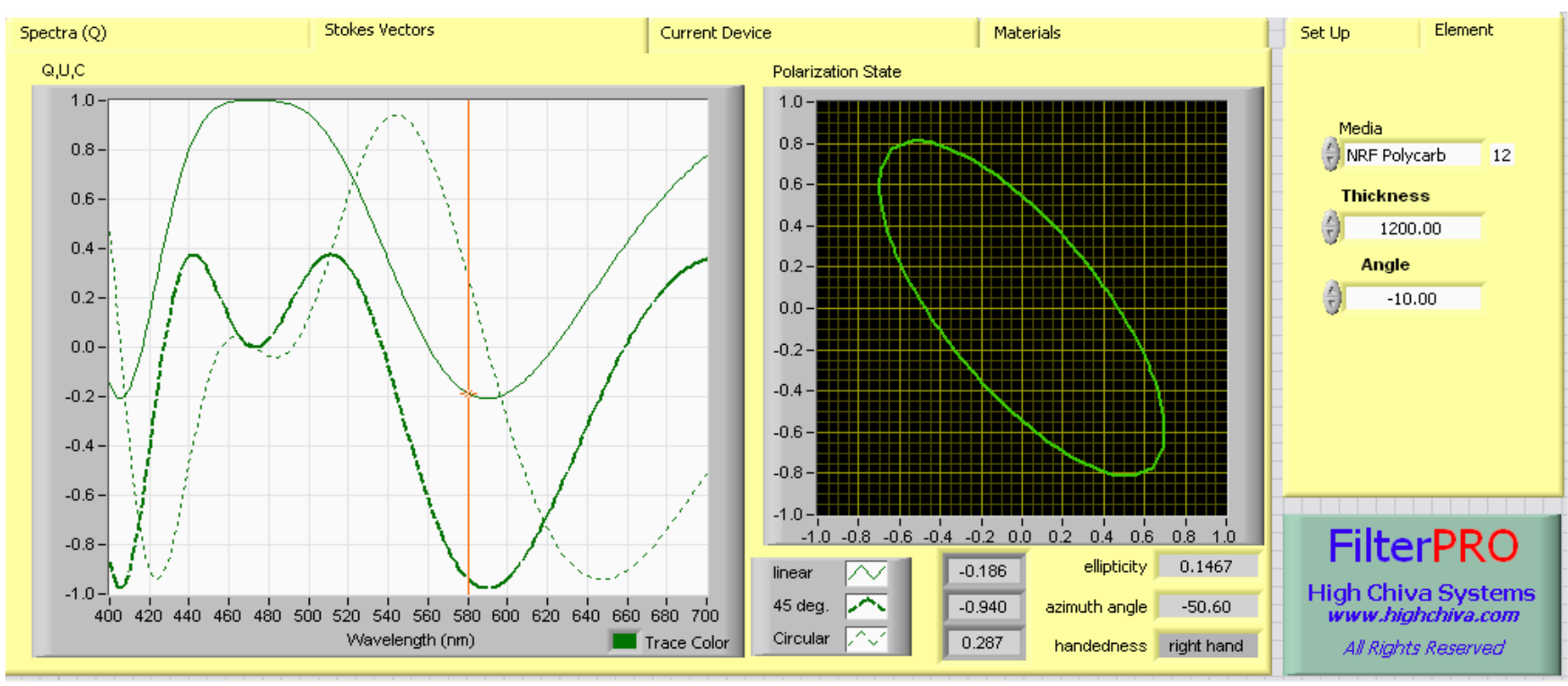

Figure 4. Details tab of FilterPRO. The user can peruse and manipulate spectral and polarization details of the current device or subcomponent.

FilterPRO was used to design the European Space agency filter that employs both ferroelectric LC cells in conjunction with numerous commercially available polymer birefringent sheets. This filter has essentially 6 usable states; 3 narrowband and 3 wideband. The target specifications are given in Table 1.

\begin{tabular}{|l|l|}
\hline \multicolumn{2}{|c|}{ Target Specifications } \\
\hline Number of active band-pass regions & 6 \\
\hline $1,2,3$ Band-pass spectral centers & $465 \mathrm{~nm}, 540 \mathrm{~nm}, 625 \mathrm{~nm}$ \\
\hline $1,2,3$ Band-pass width (nominal FWHM) & $75 \mathrm{~nm}-100 \mathrm{~nm}$ \\
\hline 4, 5, 6 Band-pass spectral centers & $465 \mathrm{~nm}, 540 \mathrm{~nm}, 625 \mathrm{~nm}$ \\
\hline 4, 5, 6 Band-pass width (nominal FWHM) & $25 \mathrm{~nm}-45 \mathrm{~nm}$ \\
\hline Usable aperture diameter & $25 \mathrm{~mm}$ \\
\hline
\end{tabular}

Table 1. ESA spectral filter target specifications 


\section{ESA Speciality Filter}

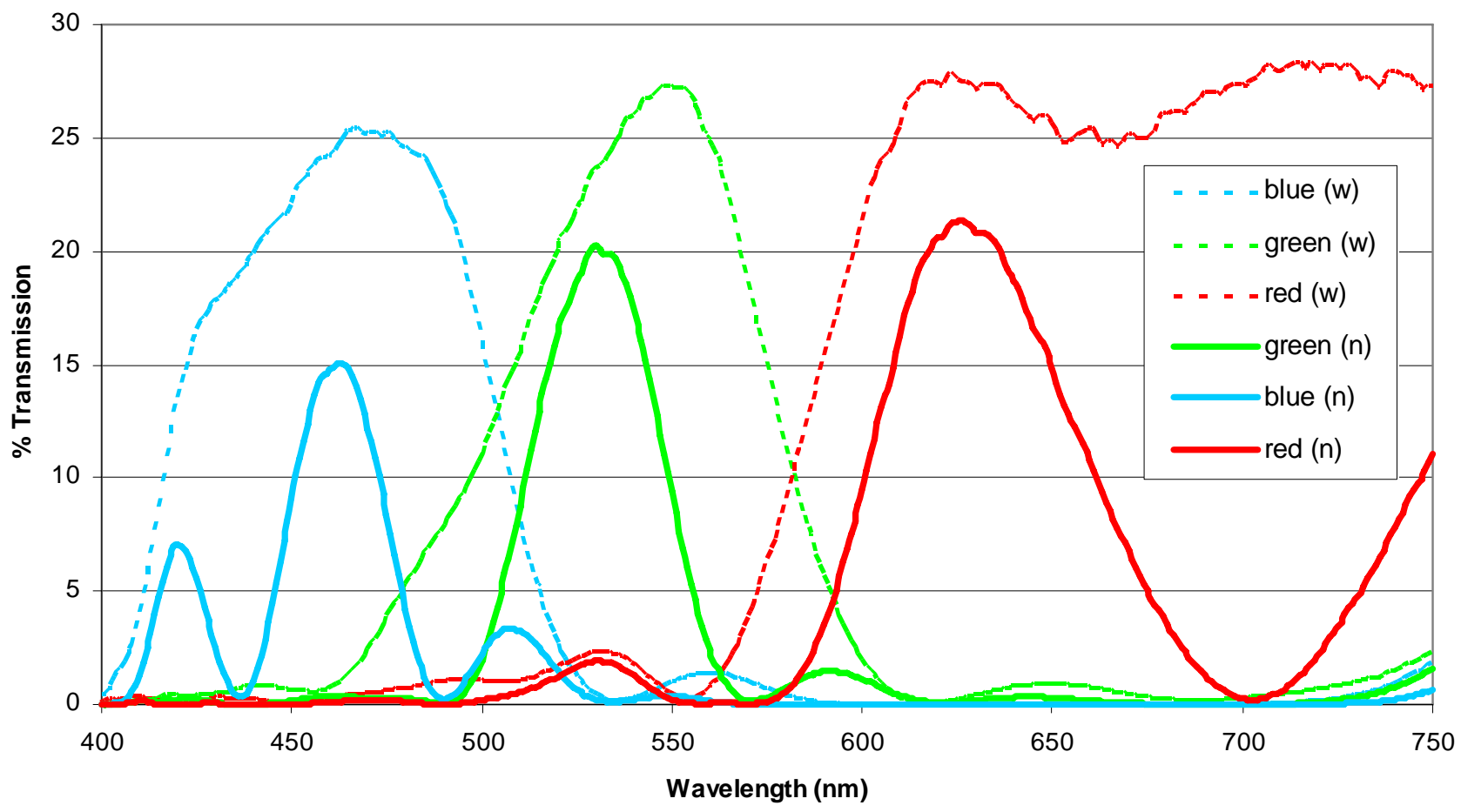

Figure5. Spectral Data from 3 stage spectral filter prototype developed for the European Space Agency. This filter was to be used with a high resolution camera for spectral imaging. The first two stages form a broadband Red-Green-Blue filter. The third stage uses a circular polarization technique and a high birefringent polymer element to narrow the spectral bands.

\subsection{Fabry Perot Etalon Array}

We are developing new class of MEMS devices for space applications using components made with highly compliant polymeric materials as a principal design element. Typically in MEMS devices, electrostatic or piezo forces are used to bend or deflect one of the MEMS layers to produce the desired mechanical motion. Stiffer materials require higher voltages to achieve a given mechanical deflection, and traditional silicon-based materials are all extraordinarily stiff. The Compliant MEMS technology platform differs from conventional MEMS by adding a set of softer, more yielding, polymeric materials to the list of conventional rigid silicon-based materials used in MEMS. Adding these compliant materials to the list of materials that can be deposited, patterned and etched greatly widens the design space. The compliant materials used in this new class of MEMS devices are as much as six orders of magnitude less stiff and can easily be tailored over a range of three orders of magnitude. Additionally, they can be deposited in a much broader range of layer thicknesses. This very wide range of flexibility expands the design space for MEMS devices far beyond what is possible with traditional silicon-based materials.

One of our Fabry-Perot components we developing is the tunable optical filter shown in figure 6. As shown in the figure an array of elastomer etalons was designed and fabricated. Each elastomer post is spaced at $1 \mathrm{~mm}$ and each column is electrically connected enabling each row of Fabry Perot Etalons to be controlled. The voltage applied to the etalon causes the distance between the mirrors, the length o the cavity L, to decrease. Since the plates of the Fabry-Perot device are moveable (i.e. it is an interferometer), then the fringes will move across the surface due to the change in constructive and destructive interference lengths. A photodiode, or photoelectric device, may be placed at the center of the interference fringes which will count the fringes as they pass by. If the distance the plate has moved by is precisely known, then it is possible to calculate the unknown 
wavelength of some source by order of interference relationship $\frac{2 L}{n}=\lambda$

wavelength of some source by order of interference relationship $n$. The variable $\mathrm{n}$ is the order of interference, or the number of fringes that pass by in our case. L represents the distance traveled by the mirror.

Another unique characteristic of Fabry-Perot Interferometer is the Free Spectral Range. This also has to do with fringe frequency, and is defined as the separation between adjacent orders of interference. This definition refers to how far the

moving mirror must move to arrive at the next order of constructive interference which occur every $\frac{n \lambda}{2}$. This relationship says that orders of constructive interference occur every half-multiple of the wavelength of the studied light. ${ }^{8}$ We are still in the process of calibrating and controlling our pixilated Fabry perot Etalon array. Figure 7 shows the spectrum from the Etalon in its relaxed state. The mirrors can be driven to $\mathrm{L}<1$ micron. We currently have no insulation layer on the electrode and due to the tile ton the mirror cannot drive it much below 5 microns due to shorting the etalon mirrors together. We are working to develop a specialized insulating layer and tilt control mechanism for the Etalon array.

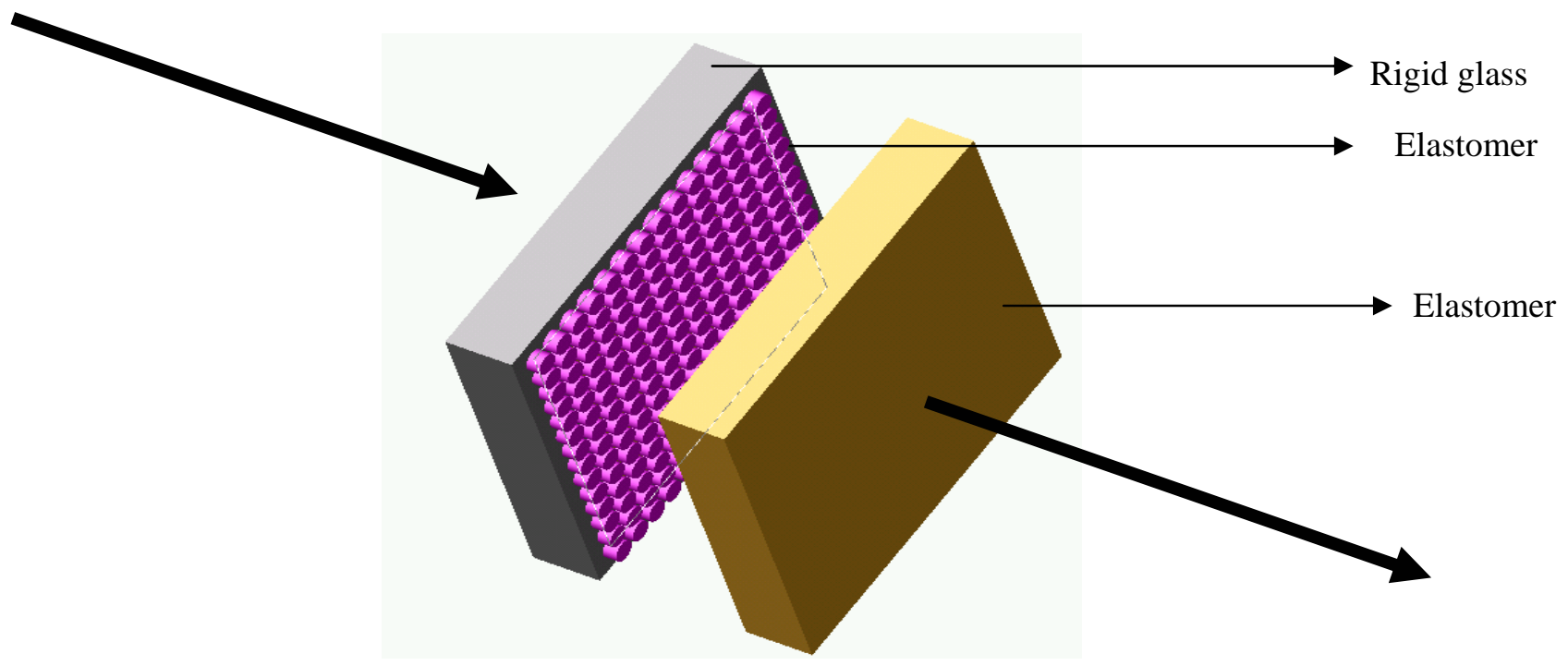

Figure 6. Schematic Elastomer Fabry Perot Etalon Array. 

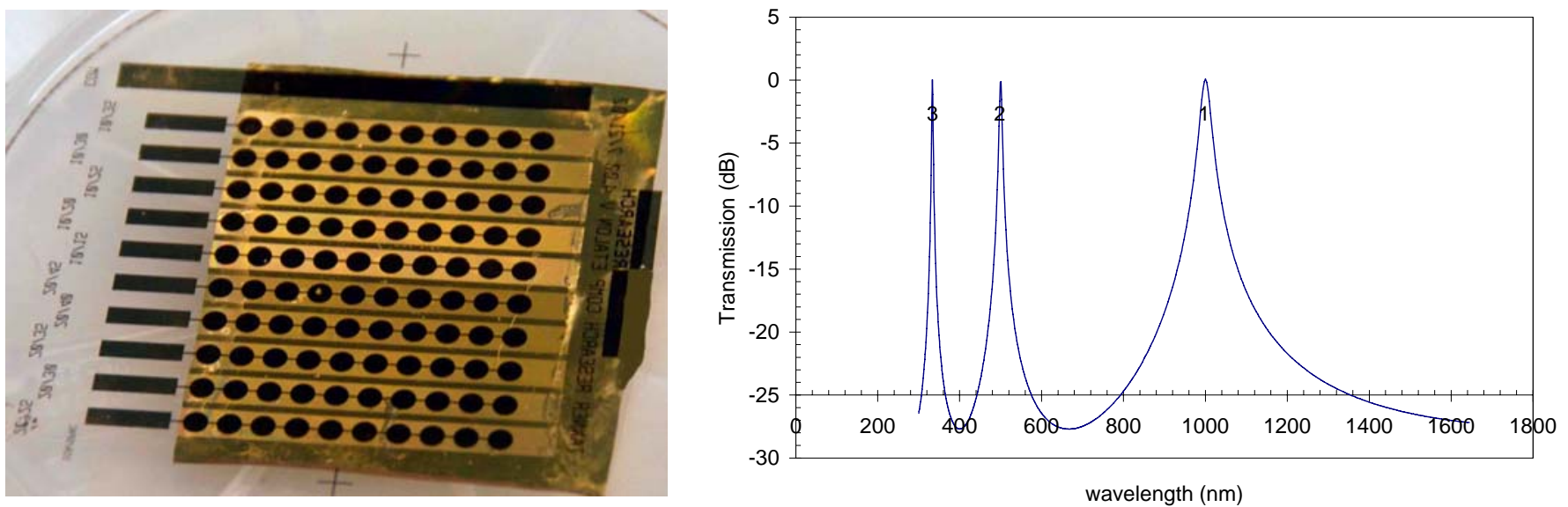

Figure 7. Fabry Perot Etalon Array.(a ) A 16x16 array of elastomer Fabry Perot and (b) its spectral transmission

\subsection{NASA Space Exploration Space Applications}

NASA plans to use the liquid crystal tunable filter in a star simulator to emulate the spectral distribution of stars for laboratory testing of an Intelligent Star Tracker (figure 8). Currently available commercial star sensors typically weigh 15 pounds, attain 5 to 10 arc-second accuracy, and use roughly 10 watts of power. Unfortunately, these commercial star sensors do not meet many of NASA's "next-generation” spacecraft and instrument needs. Nor do they satisfy Department of Defense need for micro/nano-satellite systems. NASA's Intelligent Star Tracker incorporates adaptive optic catadioptric telescope in a single, compact, robust Silicon Carbide housing. The MOEMs micro-mirrors are used to compensate for various aberrations as well as introduce aberrations such as defocus to ensure optimal system performance. The massively parallel processing architecture designed into the Intelligent Star Tracker not only enables very high bandwidths, exceeding $40 \mathrm{~Hz}$, but also enables tracking of at least 5 stars simultaneously. Moreover, the massively parallel architecture enables the Star Tracker to operate autonomously without burdening the spacecraft processor and may be used to supplement the onboard processor. Because this design utilizes technologies that inherently integrate well together and lend themselves to batch processing, we estimate that the Intelligent Star Tracker will have a recurring cost less than $\$ 100 \mathrm{k}$. We will use the tunable liquid crystal star simulator to be able to test the star tracker in our NASA laboratory under relevant space thermal and radiation conditions. Preliminary experimental result are encouraging and show our Intelligent Star Tracker system will have a pointing accuracy exceeding 0.20 arc-sec, NEA better than 0.10 arc-sec, power consumption less than $2 \mathrm{~W}$ and a weight of approximately $0.20 \mathrm{Kg}{ }^{9}$

Our tunable filters can also be used in space exploration and science mission programs that require autonomous operations that cross cut several of our NASA key technological challenge areas: (1) The Exploration Systems Architecture requires rendezvous, proximity operations, and docking (RPOD) of two spacecraft both in Low Earth Orbit (LEO) and in Low Lunar Orbit (LLO). To date, the technology readiness level of relative navigation sensors is low and has been identified as one of the Program's key risk areas. This technology leverages work performed by our partners to improve the accuracy of long-range rendezvous sensors (> 200km). (2) The Solar System Exploration Roadmap (2006) calls for real time hazard detection and avoidance systems for precision landing on other planetary bodies (e.g., Mars, Europa, Titan, etc.). In addition, future comet surface sample return missions will require the ability to safely rendezvous, descend, land, and ascend from low-gravity objects. (3) Finally, the benefits of miniaturization of hyperspectral imaging and tracking systems will apply to all 5 Flagship Class missions, in addition to Discovery and New Frontiers missions. 


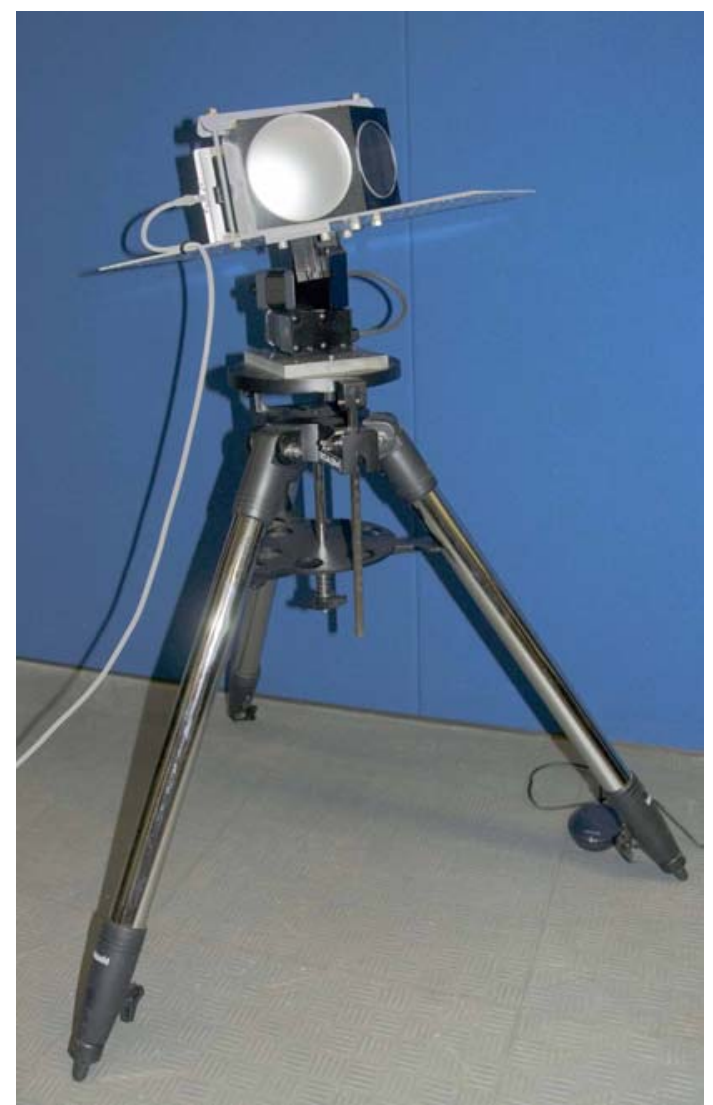

(a)

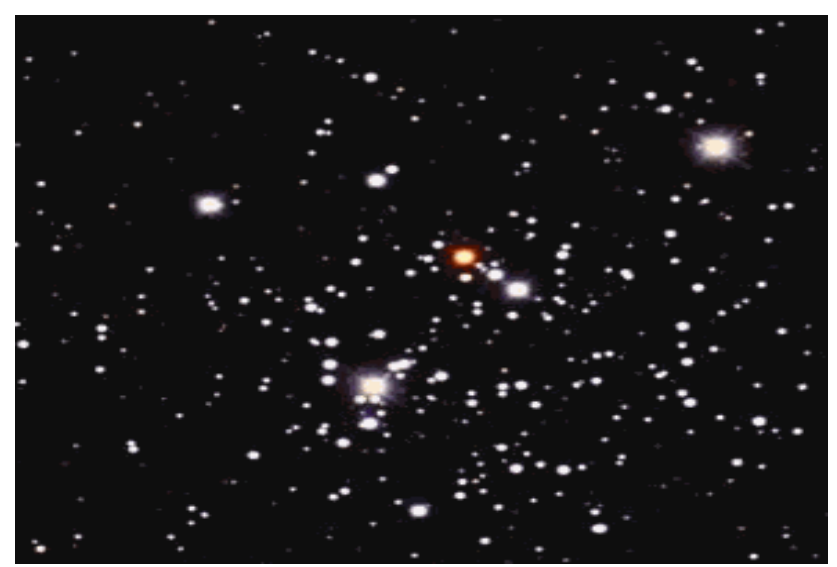

(b)

Figure 8. IntelliStar system. (a) IntelliStar mounted on a motor stage on a tripod (b IntelliStar) image of the Southern Cross using a Kodak 401 E

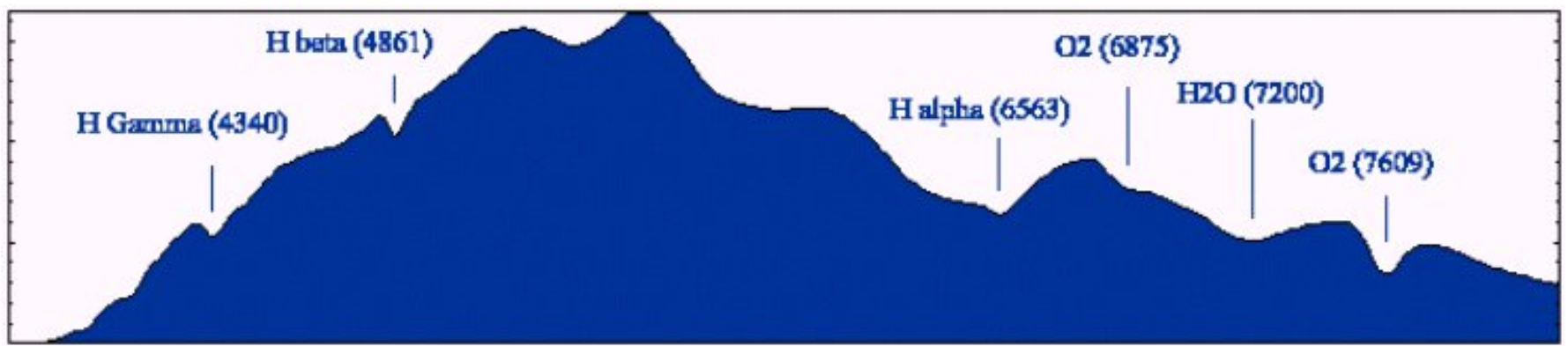

Figure 9. The spectrum of the star Vega. The blue part of the spectrum is on the left, the red part is on the right. The principal spectral lines are marked. One notices the particularly clear hydrogen series in stars of type A0. The infra-red part is made conspicuous by the presence of the molecular bands of gas components of the Earth's atmosphere: band B of O2 between 6850 and 7020 A, band H20 between 7000 and 7400 A, and band A of O2 between 7580 and 7750 A 


\subsection{Summary.}

Tunable filters play in important role in many optical systems. Our LaRC cleanroom has the capability of fabricating a wide range of tunable optical filters. Fabry-Perot filters generally fall into one of two basic categories. In the first, the distance between the parallel plates is controlled to tune the wavelength that will pass through the filter. Piezo control is most typical. Newer interferometers have employed silicon MEMS technologies to control the separation between the plates. This type of device typically has difficulty maintaining the necessary tight tolerance on the parallel orientation of the plates. Doing so is complex and expensive. Furthermore, piezo devices, in addition to requiring high voltages, have a relatively narrow operating temperature range. The voltage and thermal requirements of the piezo-electric devices impose difficult and labor-intensive manufacturing and assembly operations, adding cost to the devices and making them less reliable. The second category of Tunable filters uses fixed parallel plates and tunes the refractive index of an electro-optic material that fills the cavity between the plates; when voltage is applied, the refractive index changes. Birefringent tunable filters are one such technology we are developing for use in Space Exploration programs.

\section{References}

1. A. M. Title and W. J. Rosenberg, Opt. Eng. 20, 815 (1981).

2. I. Solc, "Birefringent Chain Filters," Journal of the Optical Society of America, 55, 621 (1965).

3. S. E. Harris, E. O. Ammann, and I. C. Chang, J. Opt. Soc. Am. 54,1267 (1964).

4. W. J. Carlsen and C. F. Buhrer, Electron. Lett. 23,106 (1987).

5. Evans, J. W., J. Opt Soc. Am 48 142(1958).

6. H. Wright, C. Crandall \& P. Miller, Active filters enable color imaging, Laser Focus World, May 1996.

7. P.D. Hale, G.W. Day, Applied Optics Vol. 27, No.24, 5146 (1988).

8. G. Hernandez, "Fabry Perot Interferometers, Cambridge: Cambridge University Press ISBN 0521322383

9 N. Clark, P. Furth, and S. Horan, “Intelligent Star Tracker”, 14th Annual/USU Conference on Small Satellites, Logan, Utah, August 2000. 